\title{
Molecular dynamics of ferroelectric polymeric systems as studied by incoherent quasielastic neutron scattering
}

\author{
E. López Cabarcos \\ Departamento de Química Fisica II, Facultad de Farmacia, Universidad Complutense, Madrid 28040, Spain
}

F. Batallán

Instituto de Ciencia de Materiales de Madrid del Consejo Superior de Investigaciones Científicas, Serrano 144, Madrid 28006, Spain

B. Frick

Institut Laue-Langevin, 38042 Grenoble Cedex, France

T. A. Ezquerra and F. J. Baltá Calleja

Instituto de Estructura de la Materia del Consejo Superior de Investigaciones Científicas, Serrano, 119, Madrid 28006, Spain

(Received 10 May 1994)

\begin{abstract}
We have studied by incoherent quasielastic neutron scattering the molecular dynamics of the ferroelectric copolymers of vinylidene fluoride and trifluoroethylene with two different molar ratios. Thermal hysteresis effects accompanying the structural changes observed in these materials are found. In the temperature range investigated three motions were identified: (i) a vibrational motion of DebyeWaller type which shows increasing amplitude until the vicinity of the Curie temperature and then it follows a $Q^{4}$ law, (ii) a slow jump diffusive motion of each chain in the restricted volume defined by other chains, thermally activated with an activation barrier of $\approx 8 \mathrm{kcal} / \mathrm{mol}$, and (iii) a fast local diffusive motion with only some fraction of the chains concerned.
\end{abstract}

\section{INTRODUCTION}

The possibility of obtaining ferroelectricity in high molar mass materials has attracted much interest in the past years. $^{1,2}$ In particular, a field of growing development is the combination of ferroelectric properties with the ductile mechanical properties of these light polymeric materials. Copolymers based on vinylidenefluoride $\left(-\mathrm{CH}_{2}-\right.$ $\mathrm{CF}_{2^{-}}$) randomly copolymerized with trifluoroethylene $\left(-\mathrm{CHF}-\mathrm{CF}_{2}-\right)$ render a semicrystalline system in which the crystalline portion of it exhibits ferroelectricity. ${ }^{3-6}$ The microstructure of these copolymers consists of stacks of lamellar crystals separated by amorphous regions. The crystalline part suffers a phase transition of the orderdisorder type below the melting temperature provided the vinylidene fluoride $\left(\mathrm{VF}_{2}\right)$ molar fraction lies below $82 \% .^{5}$ The ferroelectric crystalline phase is characterized by a pseudohexagonal structure (orthorhombic group $\mathrm{mm} 2$ ) with parallel chains in all-trans planar conformation. Upon heating the copolymers exhibit a Curie temperature $T_{C}$ at which the ferroelectric crystals show a reversible solid-state structural transformation to a disordered centrosymmetric phase (hexagonal group $6 / \mathrm{mmm}$ ). This transition has been found to involve intramolecular changes of the orientation of the dipole moments associated to the $\mathrm{CF}_{2}$ groups, which become orientally disordered around the chain axis in the high-temperature phase, and is accompanied by large enthalpy changes of about $30 \mathrm{~J} / \mathrm{g}$. The Curie temperature increases with the $\mathrm{VF}_{2}$ molar content and when compositions of about $82 \%$ are reached the crystals melt before the ferroelectric tran- sition occurs. ${ }^{5,6}$ Moreover, in the amorphous regions a glass transition is observed below $T_{C}$ for all the whole range of $\mathrm{VF}_{2}$ molar content. ${ }^{7}$ The phase diagram for these copolymers as a function of the vinylidenefluoride molar fraction has been determined by $x$-ray diffraction, ${ }^{7,8}$ dielectric $^{9}$ and calorimetric ${ }^{5,7}$ techniques, and is shown in Fig. 1.

In this paper we report new incoherent quasielastic neutron-scattering (IQNS) experimental results on the molecular dynamics of vinylidenefluoridetrifluoroethylene $\left(\mathrm{VF}_{2} / \mathrm{F}_{3} \mathrm{E}\right)$ ferroelectric copolymers in a broad temperature range covering all the observed transi-

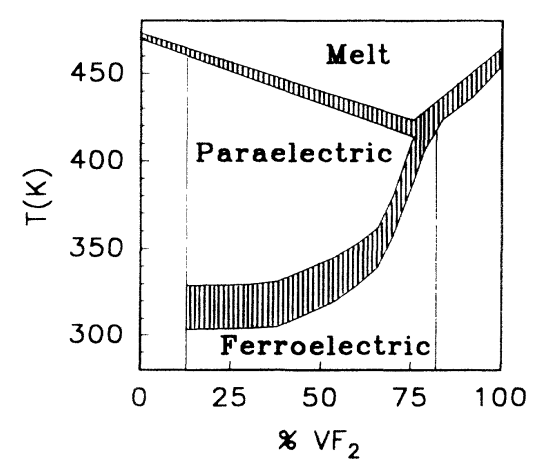

FIG. 1. Phase diagram showing the temperature vs the copolymer composition. The large transition regions among the different phases are indicated in the figure by dashed lines and depend on sample preparation as well as on copolymer thermal and processing history. 
tions. IQNS has been shown to be a powerful tool when dealing with the dynamics of polymeric systems. The basic reason for this is that the wavelength and energy of thermal neutrons are comparable to the wavelengths and energies of the fluctuations ocurring at phase transitions. Moreover, although $\mathrm{x}$-ray-scattering techniques may provide better wave-vector resolution, the energy changes can only be measured in neutron-scattering experiments. Until now the majority of the dynamic studies in polymers have been devoted to characterizing the glass transition in glass-forming polymers. ${ }^{10,11}$ In some other studies the validity of the mode coupling theory has been checked ${ }^{12}$ and some of its predictions confirmed. However, transitions ocurring in the crystalline portion (crystallinities between 0.6 and 0.8 ) of semicrystalline polymers, like the ferroelectric to paraelectric transition of $V F_{2}-F_{3} E$ copolymers, have not yet been explored in a systematic way..$^{13-15}$ On the other hand, the IQNS investigation of semicrystalline polymers allows us to study the influence of crystallinity on the dynamics of the segmental motion above the glass transition temperature. The investigation was performed on these copolymers for two reasons. This was done first, because the dynamical processes appearing in these systems are very rich and include, in order of increasing temperature, (i) the glass transition, occurring in the amorphous portion, (ii) the ferroelectric to paraelectric transition, occurring in the crystalline phase, and (iii) the melting of the crystals. At this point our aim is to separate the dynamics of the amorphous $(\alpha$ and $\beta$ relaxation processes) from that of the crystalline portions and to investigate mesoscopic time scale motions associated with the ferroelectric phase transition. Second, because the structure of these copolymers, in all their phases, has been well characterized, ${ }^{16-20}$ it is possible to correlate geometrical parameters derived from IQNS analysis with structural features.

\section{EXPERIMENTAL}

Experiments were carried out in samples with $60 / 40$ and $80 / 20\left(\%\right.$ molar $\left.\mathrm{VF}_{2} / \mathrm{F}_{3} \mathrm{E}\right)$ composition. In Table I we summarize the characteristic temperatures for both copolymer compositions: $T_{g}$ is the glass transition temperature, $T_{C}$ the Curie transition temperature, and $T_{m}$ the melting temperature. The copolymer $60 / 40$ displays an 87-K interval between $T_{C}$ and $T_{m}$, where the paraelectric phase is observed, whereas for the $80 / 20$ copolymer $T_{C}$ and $T_{m}$ are close to each other $(\Delta T=10 \mathrm{~K})$. These temperatures depend on thermal treatment. Therefore, the values given in Table $I$ are average values, which may vary depending on sample preparation as shown in Fig. 1.

TABLE I. Glass transition temperature $T_{g}$, Curie transition temperature $T_{C}$, and melting temperature $T_{m}$ of the copolymers investigated.

\begin{tabular}{llll}
\hline \hline Composition & $T_{g}(\mathrm{~K})$ & $T_{C}(\mathrm{~K})$ & $T_{m}(\mathrm{~K})$ \\
\hline $60 / 40$ & $243^{(7)}$ & $340^{(20)}$ & $427^{(20)}$ \\
$80 / 20$ & $223^{(7)}$ & $413^{(19)}$ & $423^{(19)}$ \\
\hline \hline
\end{tabular}

The neutron measurements were carried out at the Institute Laue-Langevin in Grenoble, France. In order to determine a dynamical range as large as possible, we have performed experiments using three spectrometers of different resolution. The data were collected with the backscattering spectrometers IN10 and IN13 and the time-of-flight (TOF) instrument IN6. The resolution of these spectrometers allows us to look for dynamics as slow as $10^{-9} \mathrm{~s}$ on IN10, $10^{-10} \mathrm{~s}$ on IN13 and $10^{-11} \mathrm{~s}$ on IN6. The experimental $Q$ range of the instruments was $Q=0.2-2 \AA^{-1}$ for IN10 and IN6 and $Q=0.3-5.3 \AA^{-1}$ for IN13. Measurements were made at several $Q$ vectors covering the whole $Q$ range of every instrument. The elastic backscattering study was performed for both copolymers in the full temperature range $(4-450 \mathrm{~K})$. The quasielastic spectra of the $60 / 40$ and $80 / 20$ copolymers were reecorded with both backscattering spectrometers. In addition, the $80 / 20$ copolymer was studied with the time-of-flight spectrometer IN6. The quasielastic measurements were made at selected temperatures in the ferroelectric and paraelectric phases and near the melting point. Sample films were prepared by compression moulding in a hot press at $473 \mathrm{~K}$ for $10 \mathrm{~min}$ and subsequent quenching to room temperature. Thicknesses of $450 \mu \mathrm{m}$ for $60 / 40$ and $330 \mu \mathrm{m}$ for $80 / 20$ were respectively chosen to obtain high neutron transmission $(89 \%$ and $91 \%$, respectively) and avoid multiple-scattering effects.

The experimental resolution was obtained from a measurement performed at $2 \mathrm{~K}$, where it is assumed that there are no molecular motions. The spectrum of a vanadium sample with the same shape and thickness as the polymer was also recorded to ensure the goodness of the resolution function. The spectrum of an aluminum sample holder was also registered, and background corrections were performed in all measured intensities.

\section{INCOHERENT SCATTERING FUNCTION FOR POLYMERIC SYSTEMS}

From neutron-scattering measurements we obtain the partial differential cross section $d^{2} \sigma / d \Omega d E$, which yields the portion of neutrons scattered in a given direction with a given energy. This quantity is related to the coherent and the incoherent scattering functions, $S_{\text {coh }}(Q, \omega)$ and $S_{\text {inc }}(Q, \omega)$, respectively, through the expression $^{21}$

$$
\frac{d^{2} \sigma}{d \Omega d E}=\sigma_{\mathrm{coh}} S_{\mathrm{coh}}(Q, \omega)+\sigma_{\mathrm{inc}} S_{\mathrm{inc}}(Q, \omega),
$$

where $\sigma_{\text {coh }}$ and $\sigma_{\text {inc }}$ are, respectively, the coherent and incoherent cross sections for the nucleus-neutron scattering process. The incoherent scattering function is related by means of Fourier transformation in space and time to the van Hove correlation function $G_{s}(r, t)$, which gives the probability that if a particle is at a given point at an arbitrary time, the same particle will be after a time $t$ at a distance $r$. In a similar way, $S_{\text {coh }}(Q, \omega)$ is the double Fourier transform of $G(r, t)$ which gives the probability that if a particle is at a given point at a given time, another particle, eventually the same, will be at a distance $r$ after a time $t$. 
In our samples the incoherent scattering cross section of $H$ is very large in comparison with other coherent and incoherent scattering cross sections of the atoms in the copolymers such as $C, F$, and $H$. The ratio between the incoherent scattering cross section of $H, \sigma_{i}$, and the total scattering cross section $\sigma_{t}$ is $\sigma_{i} / \sigma_{t}=0.9$. Furthermore, in the quasielastic region $S_{\text {inc }}(Q, \omega)$ has structure around $\omega=0$ for every $Q$ value. However, $S_{\mathrm{coh}}(Q, \omega)$ presents structure at $\omega=0$ only for selected $Q$ values, corresponding to the Bragg peaks, and at $\omega \neq 0$ around these peaks as collective excitations like the acoustic phonons. Provided these regions in $Q$ are avoided, the scattered intensity is almost entirely incoherent. Then, from $d^{2} \sigma / d \Omega d E$ measurements we obtain $S_{\text {inc }}(Q, \omega)$ for the $H$ atoms of the polymeric chain and hence, we can infer the overall motion of the chains.

In order to analyze the quasielastic neutron-scattering data, basically there are two alternatives. The first one takes into account phenomenological expressions such as the Kohlrausch-Williams-Watts function in the time domain or the Havriliak-Negami relaxation function in the frequency domain. These empirical functions contain some parameters, which can be fitted to the experimental results. The second approach considers geometrical models in order to simulate the dynamics of the protons attached to the polymeric chains. We have used the second approach because the scenario where the dynamics occurs has been well characterized by $x$-ray-scattering techniques.

A general motion is formed by the combination of vibrational, rotational, and translational motions. If these individual motions are uncoupled we have

$$
S_{\mathrm{inc}}(Q, \omega)=S_{\mathrm{inc}}^{\mathrm{vib}}(Q, \omega) \otimes S_{\mathrm{inc}}^{\mathrm{rot}}(Q, \omega) \otimes S_{\mathrm{inc}}^{\mathrm{tran}}(Q, \omega),
$$

where $S_{\text {inc }}^{\text {vib }}, S_{\text {inc }}^{\text {rot }}, S_{\text {inc }}^{\text {tran }}$ are the incoherent scattering functions of the vibrational, rotational, and translational motions, respectively, and $\otimes$ means convolution in the $\omega$ variable. In a polymer the situation is more complex. The vibrational motion can be consider uncoupled from other motions and is approximated to

$$
S_{\text {inc }}^{\mathrm{vib}}(Q, \omega)=\exp \left(-\left\langle u^{2}\right\rangle Q^{2}\right) \delta(\omega) .
$$

This motion is the elastic Debye-Waller factor, where $\left\langle u^{2}\right\rangle$ is the mean-square amplitude of the proton vibration. In order to describe the time dependence for the other motions we propose a quasielastic function $S_{\text {inc }}^{q}(Q, \omega)$ given by a simple Debye relaxation process in a restricted volume. Then we have

$$
S_{\mathrm{inc}}^{q}(Q, \omega)=F_{0}(Q) \delta(\omega)+\left[1-F_{0}(Q)\right] L[\omega, \Gamma(Q)],
$$

where $L[\omega, \Gamma(Q)]$ is a Lorentzian of half width at half maximum (HWHM) $\Gamma(Q)$ and $F_{0}(Q)$ the Fourier transform of the restricted volumen. The simple Debye relaxation process implies for $S_{\mathrm{inc}}^{q}(Q, \omega)$ a Lorentzian quasielastic component and the restricted volumen an elastic component. In this model we have two free functions $F_{0}(Q)$ and $\Gamma(Q)$. Provided that the inelastic contribution was separated from the quasielastic one, we have for $S_{\text {inc }}(Q, \omega)$ the general equation

$$
\begin{aligned}
S_{\text {inc }}(Q, \omega)=e^{-\left\langle u^{2}\right\rangle Q^{2}}\{ & F_{0}(Q) \delta(\omega) \\
& \left.+\left[1-F_{0}(Q)\right] L[\omega, \Gamma(Q)]\right\}
\end{aligned}
$$

that will be used to analyze our data. For the data treatment we made the convolution of this theoretical model with the experimental resolution in order to fit the experimental results and to obtain the free functions of the model. In a second step we will analyze more specific models for the $Q$ dependence of both functions in order to obtain the geometrical parameters of the dynamics.

\section{RESULTS}

\section{A. Elastic backscattering measurements}

The incoherent elastic neutron-scattering function $S_{\text {inc }}(Q, \omega=0)$, normalized to its value at $2 \mathrm{~K}$, $S_{\text {inc }}(Q, \omega=0)_{2 \mathrm{~K}}$ was measured as a function of temperature during a heating and cooling cycle with the neutron backscattering spectrometer IN10. We have used the socalled "fixed elastic window" method in which the monochromator and the analyzer are set to the same energy. Only those neutrons that change their energy by an amount smaller than the energy resolution of the spectrometer are detected. At low temperature there are only few vibrational motions and all the scattered intensity passes through the "fixed window." If some relaxational motions are excited, the elastic line broadens and only the central part within the fixed window can pass through the instrument and the observed intensity will drop. If the elastic part is well separated from the inelastic one. $S_{\text {inc }}(Q, \omega=0)$ is a good measure for the elastic scattering.

The measured incoherent elastic neutron-scattering functions for both copolymers as a function of temperature during a heating and cooling cycle are shown in Fig. 2. The intensity decreases with increasing temperature

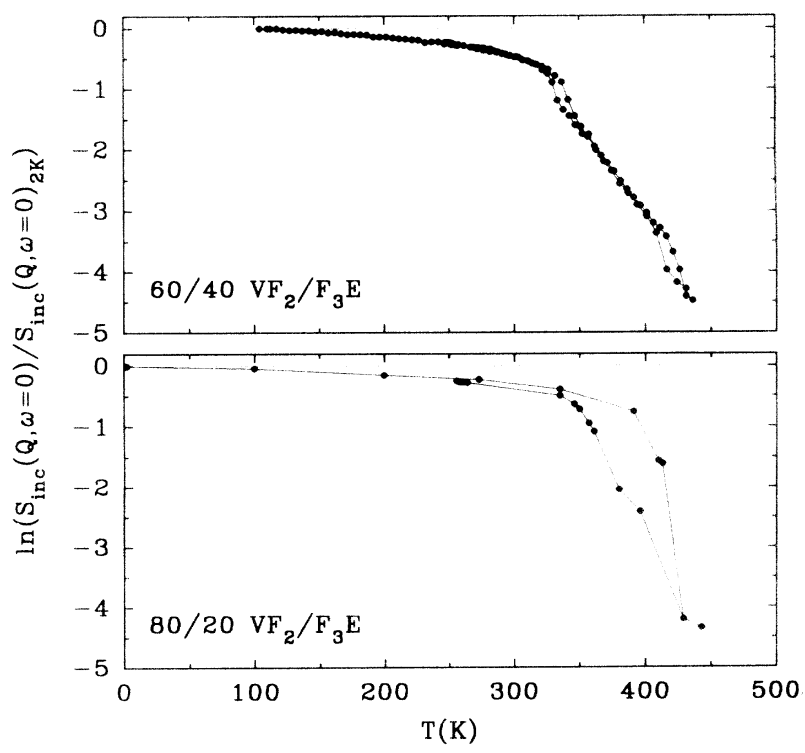

FIG. 2. Plot of $\ln \left[S_{\text {inc }}(Q, \omega=0) / S_{\text {inc }}(Q, \omega=0)_{2 \mathrm{~K}}\right]$ as a function of the temperature for the heating and cooling cycles of $60 / 40$ and $80 / 20 \mathrm{VF}_{2} / \mathrm{F}_{3} \mathrm{E}$ copolymers at $Q=1.93 \AA^{-1}$ using the "fixed elastic window" method. 


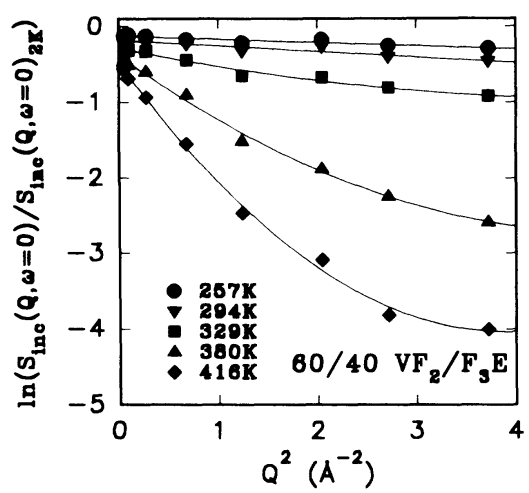

FIG. 3. Logarithm of the normalized elastic incoherent scattering function from IN10 backscattering spectrometer vs $Q^{2}$ for $60 / 40$ copolymer. The data are fitted by straight lines below $T_{C}$ to obtain the mean-square displacement $\left\langle u^{2}\right\rangle$.

and decreasing $Q$ (Fig. 3). For the $60 / 40$ copolymer four regions can be identified: (i) In the temperature region below $T_{g}$ the normalized intensity decreases linearly with $T$. (ii) For $T>T_{g}$ the rate of intensity decrease becomes slightly larger, far beyond of what is expected from an harmonic behavior. (iii) At the Curie temperature a pronounced additional intensity decrease occurs. Here the $\ln \left[S_{\text {inc }}(Q, \omega=0) / S_{\text {inc }}(Q, \omega=0)_{2 \mathrm{~K}}\right]$ versus $T$ follows a linear behavior until the vicinity of the melting point. (iv) On approaching the melt temperature a new variation of the slope appears.

In a heating-cooling cycle around the transition temperatures, two hysteresis loops can be observed for the $60 / 40$ copolymer. They correspond to the thermal hysteresis of this copolymer at the ferroelectric-paraelectric and paraelectric-liquid phase transitions. The sample with composition $80 / 20$ shows a similar behavior but with only one hysteresis loop. In this copolymer the $\mathrm{Cu}$ rie and melt temperatures are very close and consequently we observe the superposition of both hysteresis loops as confirmed by differential-scanning-calorimetry (DSC) measurements. ${ }^{15,20}$

As we have

$$
\ln \frac{S_{\mathrm{inc}}(Q, \omega=0)}{S_{\mathrm{inc}}(Q, \omega=0)_{2 \mathrm{~K}}}=\left[\left\langle u^{2}(2 \mathrm{~K})\right\rangle-\left\langle u^{2}(T)\right\rangle\right] Q^{2},
$$

from the elastic intensity we obtain the temperature dependence of the mean-square vibrational amplitude $\left\langle u^{2}(T)\right\rangle$ of the Debye-Waller factor (DWF). This regime implies a spatial dependence in $Q^{2}$. Furthermore, a linear variation of $\left\langle u^{2}(T)\right\rangle$ with $T$ corresponds to a harmonic behavior.

The first deviation from linearity arises around the glass transition temperature in the amorphous portion of the copolymers. The spatial scale of the motion is evaluated analyzing the $Q$ dependence of the elastic intensity, which is shown in Fig. 3 for the 60/40 at several temperatures. We found that in the temperature range below $T \approx 290 \mathrm{~K}$ the $\ln \left[S_{\text {inc }}(Q, \omega=0) / S_{\text {inc }}(Q, \omega=0)_{2 \mathrm{~K}}\right]$ follows

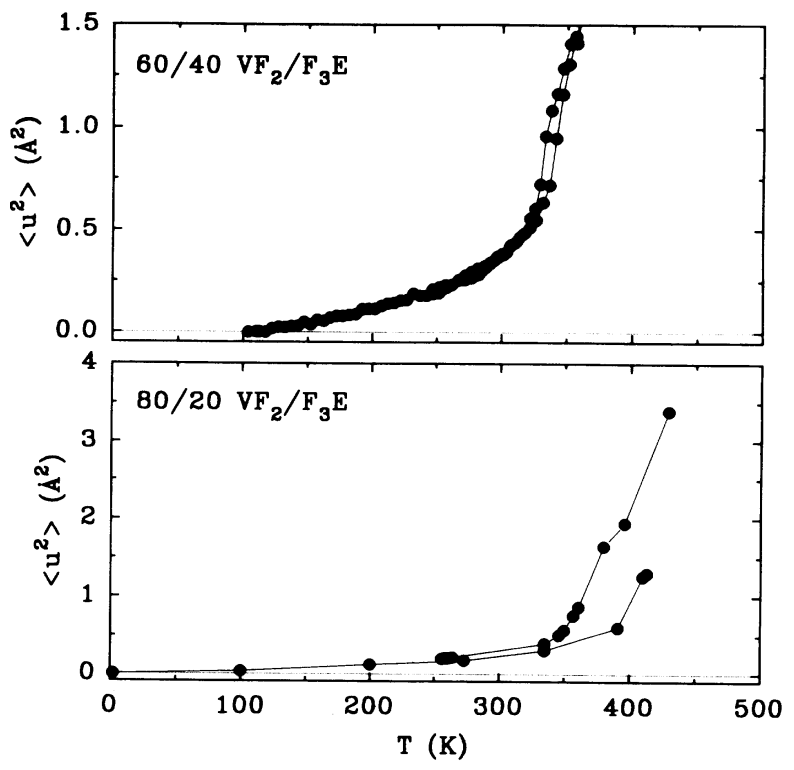

FIG. 4. Mean-square displacement as a function of the temperature for both copolymers obtained from the fitting of the IN10 backscattering data. The values obtained during a heating and cooling cycle agree below $T_{C}$.

a $Q^{2}$ law, whereas a deviation from this behavior to a $Q^{4}$ law occurs around $T_{C}$. In order to explain the latter behavior, $S_{\text {inc }}(Q, \omega)$ given by Eq. (5) should be modified to include the coupling of the motions. Such a dependence has been theoretically predicted for systems with a negative Flory interaction parameter $x$ (Ref. 22) and experimentally reported in polymer melts. ${ }^{23}$ Figure 4 shows the $\left\langle u^{2}\right\rangle$ variation with temperature for both copolymers below $T_{C}$. At low temperatures the protons are subjected to a harmonic potential and $\left\langle u^{2}\right\rangle$ follows a linear dependence with the temperature. However, at temperatures higher than $290 \mathrm{~K}$ deviations from this behavior appear due to anharmonic contributions. Similar deviations were found in amorphous polymers like polybutadiene, ${ }^{11}$ and they were not accompanied by quasielastic broadening. Fujara and Petry ${ }^{24}$ point out that the extra decrease of the elastic intensity finds its counterpart in a strong increase of the inelastic part of $S_{\mathrm{coh}}(Q, \omega)$ of the spectrum, which should appear with the IN13 spectrometer as a flat background, which increases with $Q$. The region of the ferroelectric to paraelectric phase transition appears as a clear step in the $\ln \left[S_{\text {inc }}(Q, \omega=0) / S_{\text {inc }}(Q, \omega=0)_{2 \mathrm{~K}}\right]$ versus $T$ plot. The elastic intensity swings in this case into the quasielastic component around $T_{C}$, which is observable with both IN10 and IN13 spectrometers. When the melting temperature is approached the elastic component disappears and only the quasielastic scattering is observed.

\section{B. Quasielastic backscattering}

The observed deviation of the elastic scattering from the linear behavior in the $\ln \left[S_{\text {inc }}(Q, \omega=0) /\right.$ $\left.S_{\text {inc }}(Q, \omega=0)_{2 \mathrm{~K}}\right]$ versus $T$ plot may indicate the onset of 


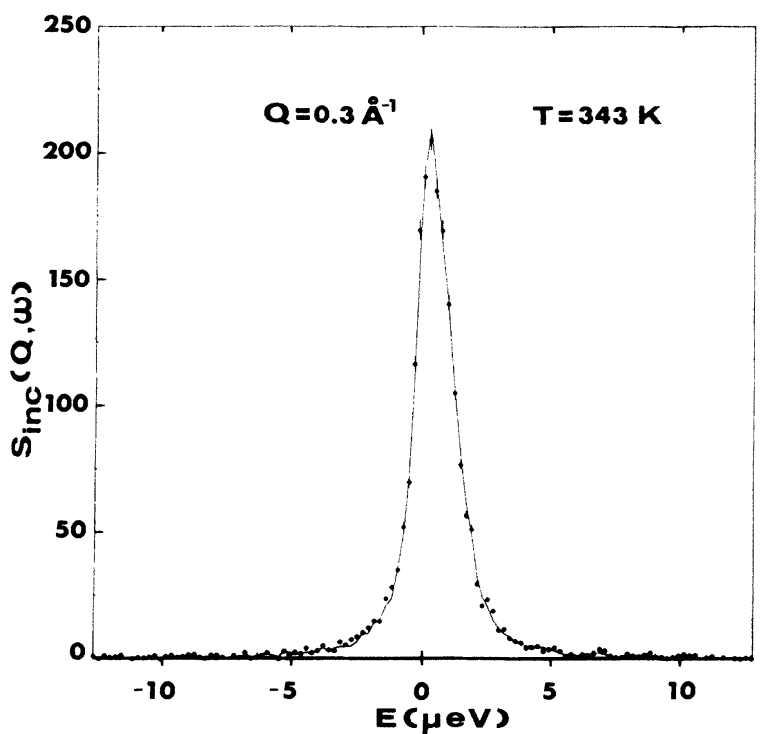

FIG. 5. The $S_{\text {inc }}(Q, \omega)$ vs energy transfer for the copolymer $60 / 40$ at $343 \mathrm{~K}$ illustrates that the response is still elastic at this temperature.

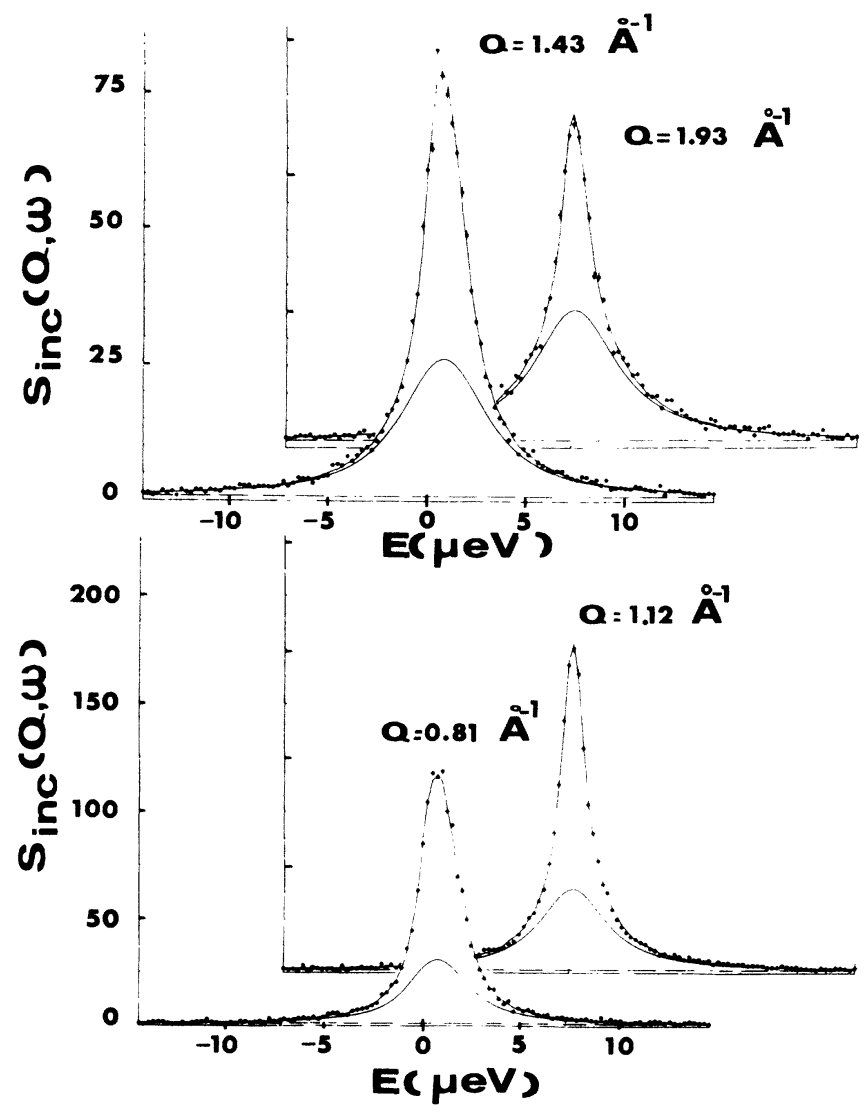

FIG. 6. Quasielastic neutron-scattering spectra as a function of $Q$ for the $60 / 40$ copolymer at $363 \mathrm{~K}$ as obtained in the IN10 spectrometer. The solid lines through the points represent the fitting results. The quasielastic component and the flat background are represented in the same figure. some molecular motion. Therefore, we have investigated the quasielastic spectrum of the $60 / 40$ copolymer in the different regions. Below the Curie temperature there is not quasielastic scattering within the dynamical range of the instruments. Even at temperatures slightly above $T_{C}$ the quasielastic component is small. As an example, we show in Fig. 5 the experimental curve at $T=343 \mathrm{~K}$ fitted with the measured instrumental resolution function convoluted with a $\delta$ function. Only in the wings is possible to appreciate some disagreement between the two curves, which points out to the onset of quasielastic broadening. If we increase the temperature beyond $T_{C}$ (paraelectric region), the quasielastic component can then be clearly observed. In Fig. 6, we show the quasielastic component at $363 \mathrm{~K}$ together with the fitting result according to Eq. (4) for several $Q$ values. Furthermore, in the temperature range corresponding to the paraelectric phase the intensity of the quasielastic component increases with temperature. Finally near the melting point the elastic part diminishes considerably and most of the intensity is quasielastic.

Although, the $80 / 20$ copolymer in some aspects behaves similarly to the $60 / 40$ it presents some particularities. As in the case of the 60/40, below the ferroelectric transition the experimental spectra can be very well fitted with the instrumental resolution function convoluted with a $\delta$ function as is illustrated in Fig. 7 for the temperature $391 \mathrm{~K}$. The onset of the motion, as indicated by the beginning of the quasielastic broadening, is around $400 \mathrm{~K}$. Figure 8 shows the experimental spectra at $403 \mathrm{~K}$ obtained from IN13 spectrometer, for several $Q$ values, together with the fitting result. The observed quasielastic scattering at $403 \mathrm{~K}$, below $T_{C}$, seems to indicate that although in the 60/40 copolymer the onset of the motion takes place at the Curie temperature, in the 80/20 the proximity of the Curie temperature to the melting point

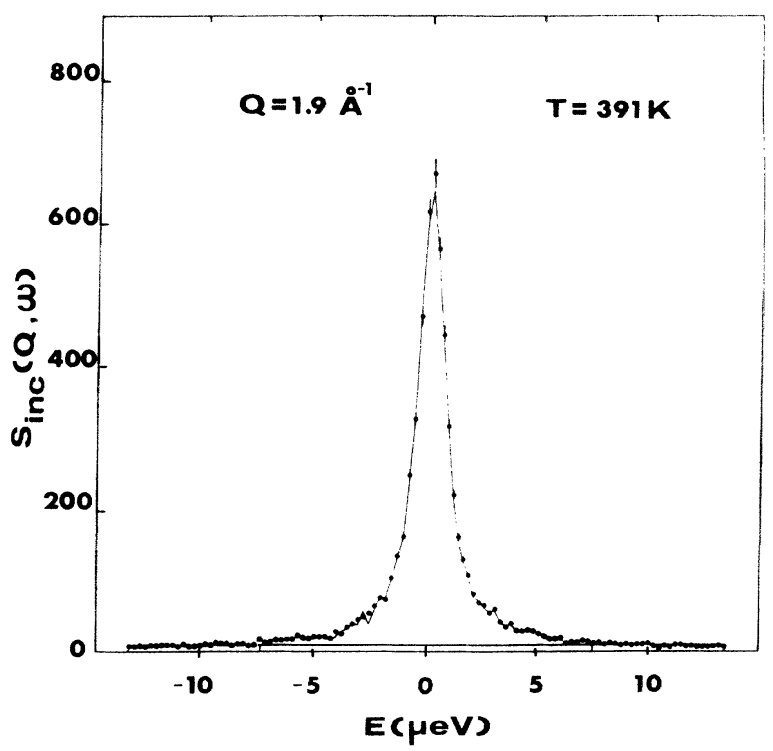

FIG. 7. The $S_{\text {inc }}(Q, \omega)$ vs energy transfer for the copolymer $80 / 20$ at $391 \mathrm{~K}$ illustrate that the response is still elastic at this temperature. 


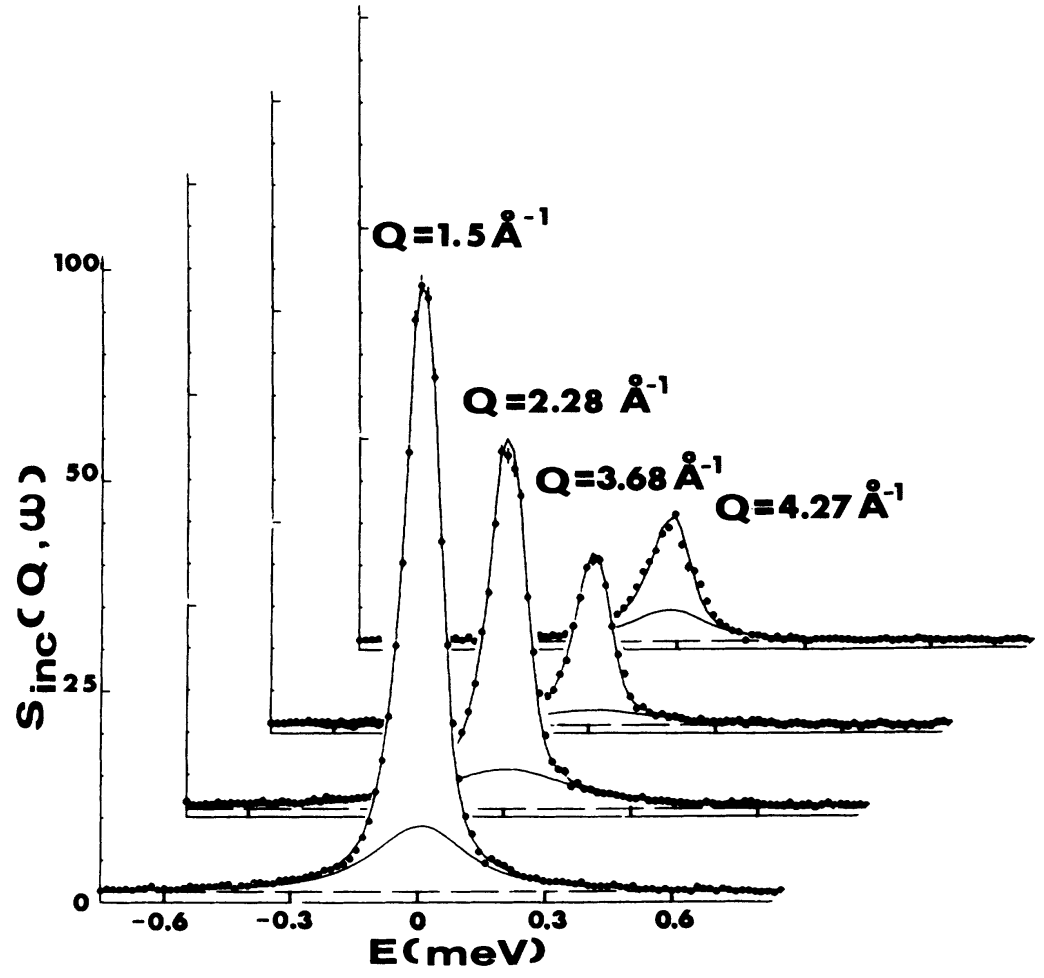

FIG. 8. Quasielastic neutron-scattering spectra as a function of $Q$ for the $80 / 20$ copolymer at $403 \mathrm{~K}$ as obtained in the IN 13 spectrometer. The solid lines through the points represent the fitting results. The quasielastic component and the background are represented in the same figure. favors the process. We have observed that at $391 \mathrm{~K}$ no quasielastic broadening was measured. It is between 391 and $403 \mathrm{~K}$ when the motion is activated.

In both spectrometers it seems clear from the tails of the experimental spectra that a flat background is present. As shown in Figs. 6 and 8 the result of the fitting was improved by introducing a flat background whose value is determined from the data of the tails of the curve. Thus, we obtained the HWHM of the quasielastic component, $\Gamma(Q)$, and the amplitude $F(Q)$. Figure 9 shows the values of the $\Gamma(Q)$ versus $Q^{2}$ obtained for the copolymer $60 / 40$ at four temperatures above $T_{C}$. In these plots we represent together the data obtained with both IN10 and IN13 spectrometers. The values of the HWHM of the quasielastic component present a continu-

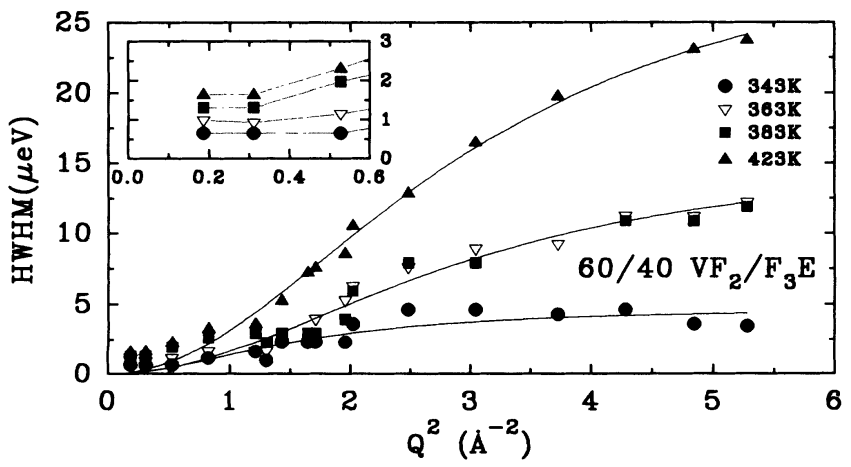

FIG. 9. HWHM of the Lorentzian quasielastic component, measured in IN10 and IN13 spectrometers, as a function of $Q^{2}$ for the copolymer $60 / 40$ at several temperatures in the paraelectric phase. ous trend from IN10 to IN13 and superimpose with each other for the $Q$ range, where both spectrometers coincide. The HWHM data for larger $Q$ values are omitted because owing to the decrease of the intensity with $Q$ there is a pour statistics for $Q^{2}$ larger than $5.5 \AA^{-2}$. Although there is a big difference at high $Q$ between the values of the HWHM for both extreme temperatures investigated, in the intermediate temperature region the differences are very small as can be seen in Fig. 9 for 363 and $383 \mathrm{~K}$. The same tendency was observed in the copolymer $80 / 20$ in which no difference in the two temperatures investigated 403 and $413 \mathrm{~K}$ was observed by comparing HWHM. The measured values for the latter copolymer at $413 \mathrm{~K}$ are shown in Fig. 10 in the full $Q$ range investigated. The anomalous behavior at $Q^{2} \approx 2$ could be attributed to the fact that this is the smallest $Q$ value measured with IN13,

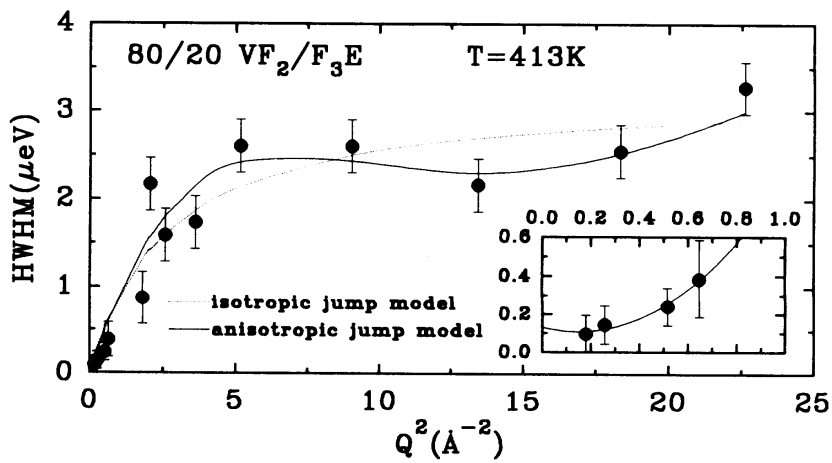

FIG. 10. HWHM of the Lorentzian quasielastic component, measured in IN10 and IN13 spectrometers, as a function of $Q^{2}$ for the copolymer $80 / 20$ at $413 \mathrm{~K}$. 
and we are within the limit of the resolution of the instrument. Although in this sample we obtained better statistics some fluctuations are observed at high $Q$ around a mean value of $2.6 \mu \mathrm{eV}$.

In a first step to analyze the experimental data of Fig. 9 we propose a isotropic jump diffusion model. When in this model the jump parameter is randomly oriented and if there are a random distribution of jump lengths centered around $r_{0}$ the HWHM is given by

$$
\Gamma(Q)=\frac{1}{\tau}\left[1-\frac{1}{1+Q^{2} r_{0}^{2}}\right]
$$

where $\tau$ is the residence time. In Fig. 9 we show the fitting result of the experimental data by this model to obtain the values of $r_{0}$ and $\tau$ for each temperature.

Although this model predicts for $Q \rightarrow 0$ that $\Gamma(Q)=0$ our results do not show such a behavior as can be seen in the insert of Fig. 9. We have interpreted this behavior in the basis of a jump diffusion model in a restricted volume. According to this model the maximum at high $Q$ implies that the motion is a jump diffusion and the nonzero value in the limit $Q \rightarrow 0$ indicates that it occurs in a restricted volume. The incoherent scattering function $S_{\text {inc }}(Q, \omega)$ for such a model has been calculated under different conditions. Volino and co-workers, ${ }^{26,27}$ have calculated the continuous diffusion in a restricted volume of simple geometrical shape as a sphere or a cylinder. Hall and Ross $^{28}$ have calculated the case of the spatially bounded random jumps in a volume limited by two boundaries (one dimension) assuming a Gaussian distribution of jump lengths. The asymptotic value $(Q \rightarrow 0)$ for both models is the same. The incoherent scattering function for the last model is given by ${ }^{21,25}$

$$
S_{\mathrm{inc}}(Q, \omega)=F_{0}(Q L) \delta(\omega)+\sum_{n=1}^{\infty} F_{n}(Q L) L[\omega, \Gamma(Q L)]
$$

the amplitude of the elastic component is

$$
F_{0}(Q L)=j_{0}{ }^{2}(Q L / 2) \text {, }
$$

where $j_{0}$ is the spherical Bessel function of first kind. The amplitudes of the Lorentzians are given by

$$
F_{n}(Q L)=\frac{(2 Q L)^{2}}{\left[(Q L)^{2}-(n \pi)^{2}\right]^{2}}\left[1-(-1)^{n} \cos (Q L)\right] .
$$

The HWHM of the Lorentzian functions $L_{n}(\omega, \Gamma)$ is expressed by

$$
\Gamma(Q L)=\frac{1}{\tau}\left[1-\exp \left(-n^{2} \pi^{2} r_{0}^{2} / 2 L^{2}\right)\right],
$$

where $L$ is the distance between two impermeable boundaries, $r_{0}$ the mean-square jump length and $\tau$ the dwell time between two successive jumps. The random-jump restricted diffusion model exhibits an asymptotic behavior at low and high $Q$. At low- $Q$ values we are observing motions extended to large distances and affected by boundary effects. In this case, $\Gamma$ tends to $\pi^{2} r_{0}^{2} / 2 L^{2} \tau$. At large $Q$ values we are looking at short distances and the $\Gamma$ values tend to $1 / \tau$. The wall separation $L$ has been evaluated from the experimental elastic incoherent

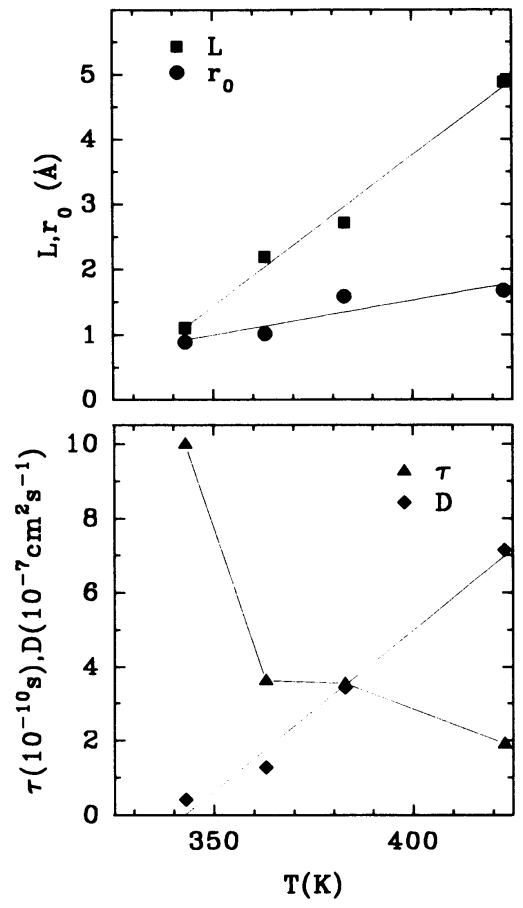

FIG. 11. Temperature variation in the $60 / 40$ copolymer of (a) jump distance $r_{0}$ and characteristic length $L$ of the available volume and (b) residence time $\tau$ and diffusion constant $D$.

scattering factor (EISF), and $r_{0}$ and $\tau$ from the experimental asymptotic values of the HWHM. Figure 11(a) shows the variation of $r_{0}$ and $L$ with the temperature. The jump length $r_{0}$ exhibits small change varying between 0.8 and $1.6 \AA$. However, the wall separation $L$ increases with temperature. At the Curie temperature (340 $\mathrm{K}), r_{0}$ and $L$ values are nearly the same suggesting that all the free available volume is occupied by the jump. At the melting temperature $(427 \mathrm{~K}) L$ is of the order of the average distance between chains that in both copolymers is closed to $5 \AA$ as measured by wide angle $\mathrm{x}$-rayscattering techniques. ${ }^{15}$ The $L$ variation with the temperature suggests that, whereas at low temperature the boundaries are given by the chain itself when the temperature increases the chain motion is limited by the neighboring chains and the $L$ behavior parallels that of the lattice spacing. ${ }^{15}$ Figure $11(\mathrm{~b})$ shows the rapid decrease of the time between successive jumps $\tau$ as a function of temperature. The diffusion coefficient $D$ in the random jump diffusion model is given by

$$
D=r_{0}^{2} / 2 \tau \text {. }
$$

The value of $D$ is plotted in the same figure. The temperature dependence of $D$ follows an Arrhenius law:

$$
D=D_{0} \exp \left(-\Delta E_{a} / k_{B} T\right)
$$

and an activation energy of $\Delta E_{a} \approx 8 \mathrm{kcal} / \mathrm{mol}$ for the copolymer $60 / 40$ was obtained. Values of $\approx 10 \mathrm{kcal} / \mathrm{mol}$, using potential energy calculations, were found in pure $\mathrm{PVF}_{2}$ and correspond to a rotation of $135^{\circ}$ around the chain axis. This result is of the same order of magnitude 


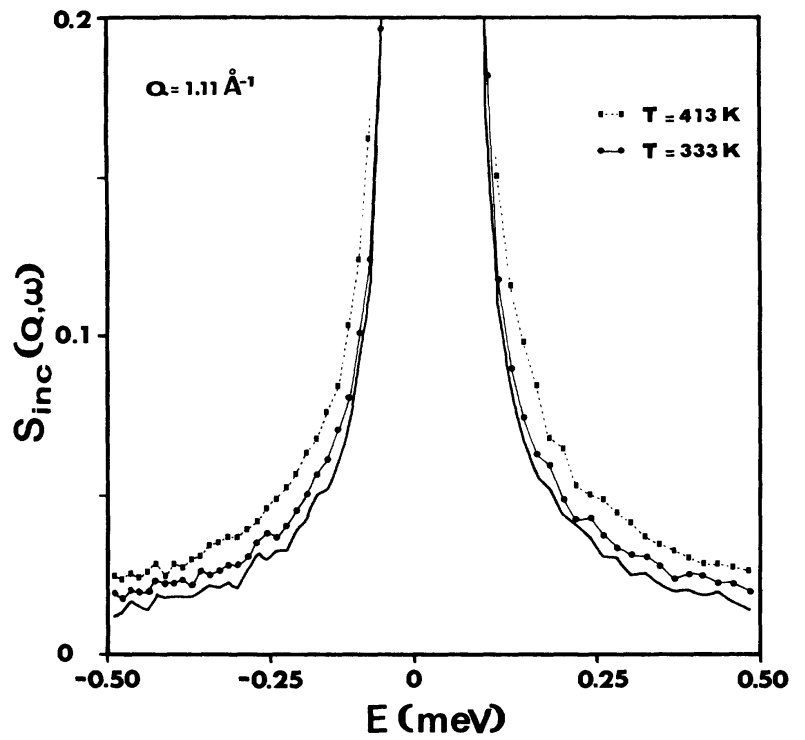

FIG. 12. Incoherent scattering function as measured by IN6 for $Q=1.1 \AA^{-1}$ at $T=333$ and $413 \mathrm{~K}$. Solid line is the instrumental resolution.

as that we have obtained and seems to indicate that this is the main contribution to the activation energy, the packing energy having only a small effect.

The behavior of the HWHM for the copolymer 80/20 is illustrated in Fig. 10 and seems to indicate that the same model could be applied for this copolymer. In this sample we show the whole $Q^{2}$ range recorded, which extend until $25 \AA^{-2}$. We have fitted the data with the isotropic random jump diffusion model according to Eq. (7). For $Q^{2}$ higher than $15 \AA^{-2}$ the model exhibits a tendency to the saturation. However, the experimental results show systematic oscillations around the saturation value, which are not explained by the previous model as denoted by the dotted line calculated with $r_{0}=0.6 \AA$ and $\tau=13 \mathrm{~ns}$. In order to take into account these oscillations we have considered an anisotropic random jump diffusion model. According to this model ${ }^{23}$

$\Gamma(Q)=\frac{1}{\tau}\left[1-\cos \left(Q r_{x}\right) / 3-\cos \left(Q r_{y}\right) / 3-\cos \left(Q r_{z}\right) / 3\right]$,

where it is assumed that the jump length can be different in the three directions in the space. In Fig. 10 we have superimposed the fitting result with this model to the experimental data and as can be seen in the figure the oscillations at high $Q$ are then well described. As a result of the fitting we have obtained the jump lengths $r_{x}=r_{y}=0.58 \AA$ and $r_{z}=1.52 \AA$ and the dwell time $\tau=9$ ns.

\section{Time-of-flight experiments}

As the flat background which increases with $Q$ and temperature (see Fig. 6), observed in IN10 and IN13 could indicate that some other motion is activated we have performed investigations in the time-of-flight spectrometer IN6. With this instrument is possible to observe energy transfers between -50 and $50 \mathrm{meV}$ with an energy resolution of $100 \mu \mathrm{eV}$. TOF experiments were carried out on the $80 / 20$ copolymer to fully explore the temperature range of existence of the ferroelectric phase, which in this sample is larger than in the 60/40. At $90 \mathrm{~K}$ there is not quasielastic broadening and the spectra can be fitted with the resolution function convoluted with a $\delta$ function. The same result was obtained at $303 \mathrm{~K}$ although at this temperature a very weak quasielastic broadening can be observed only at high $Q$ values $\left(Q=1.7\right.$ and $\left.1.9 \AA^{-1}\right)$. At $333 \mathrm{~K}$ as illustrated in Fig. 12, a weak quasielastic component can be appreciated appearing at smaller $Q$ values $\left(Q=1.1 \AA^{-1}\right)$. A clear and broad quasielastic component in the whole $Q$ range is observed at $393 \mathrm{~K}$ indicating the existence of a motion faster than the one described in the preceding section. For the initial treatment we have used the model given by Eq. (4). Figure 13 shows $F_{0}(Q)$, the EISF as a function of the momentum transfer at several temperatures upon heating. In the ferroelectric and paraelectric phases the EISF depends on the temperature. However, at higher temperatures in the melt the EISF is the same for all temperatures. This dependence of the EISF suggests that we cannot consider the same dynamics for all the proton scatterers. The simplest hypothesis is to consider two kinds of proton scatterers; a fraction $n(T)$ contributing to a diffusion motion in a restricted volume and the other $1-n(T)$ remaining without motion. Thus we generalize Eq. (4) by

$$
\begin{aligned}
S_{\mathrm{inc}}(Q, \omega)= & n(T)\left\{F_{0}(Q) \delta(\omega)+\left[1-F_{0}(Q)\right] L[\omega, \Gamma(Q)]\right\} \\
& +[1-n(T)] \delta(\omega)
\end{aligned}
$$

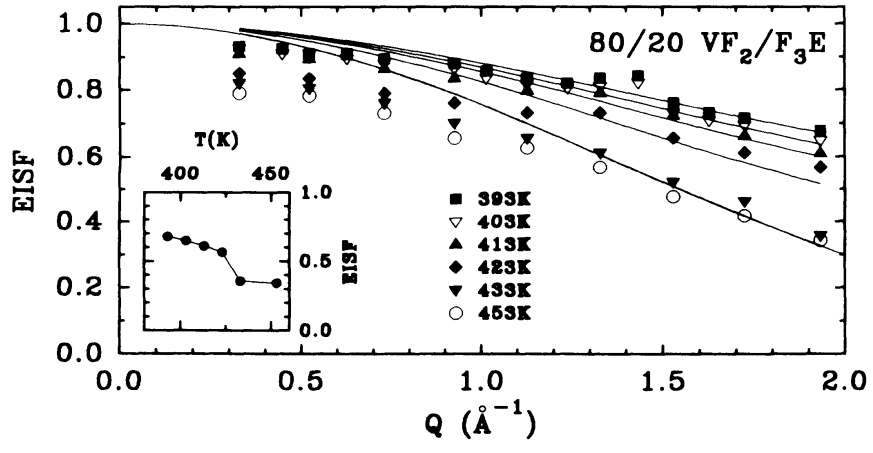

FIG. 13. Fast motion: EISF as a function of $Q$ at several temperatures for the copolymer $80 / 20$. The inset shows the temperature variation of the EISF for the highest $Q$ value. 


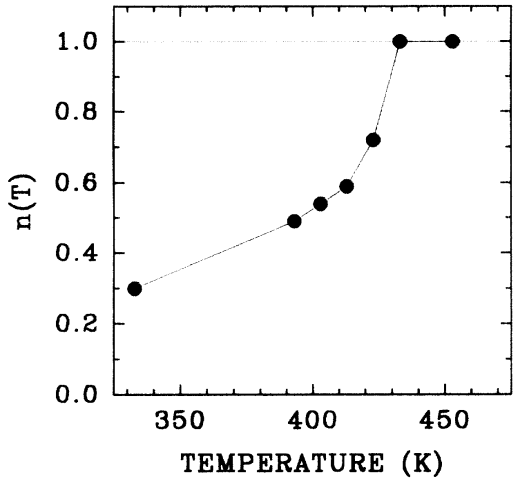

FIG. 14. Fast motion: fraction of protons in motion as a function of temperature. Above $T_{m}$ all protons are in motion. Below $T_{C}$ some protons remain in motion.

The observed EISF could then be described by the junction

$$
F_{\mathrm{EISF}}(n, Q)=n(T) F_{0}(Q)+1-n(T) .
$$

In the molten phase, where all the chains are in motion, $n(T)=1$ and the EISF is $F_{0}(Q)$ independent of $T$. In order to estimate a characterstic size, $d$, for the fast motion we assume for $F_{0}(Q)$ a rotational diffusion model in a sphere of diameter $d$. Then, we have

$$
F_{0}(Q)=j_{0}^{2}(Q d / 2) \text {. }
$$

From the fitting of $F_{0}(Q)$ to the experimental result we obtained $d=1.7 \AA$, suggesting a fast motion of small amplitude within the chain segments. As the $d$ value is of the order of the van der Waals radius of individual $\mathrm{C}$ atoms we visualize the onset of this motion as a rotation around the covalent $\mathrm{C}-\mathrm{C}$ bonds. Once $F_{0}(Q)$ is known then $n(T)$ can be obtained by imposing an homotetic behavior of the $F_{\text {EISF }}(n, Q)$. The value of $n$ shows a considerable increase with temperature as illustrated in Fig. 14. For low temperatures $T<413 \mathrm{~K}$ the agreement of the fitting with the experimental results is good even though for $Q<0.5 \AA^{-1}$ there are systematic differences, which we attribute to multiple-scattering effects. At temperatures higher than $423 \mathrm{~K}$ when the polymer is in the melt the disagreement in the low- $Q$ region increases. During the measuring time (around $6 \mathrm{~h}$ ) the polymer flows down and changes slightly its thickness given rise to an increase in the multiple-scattering effects. A crude way to correct the multiple-scattering effects is the multiplication of the experimental EISF by a constant factor determined imposing at the lowest $Q$ the same EISF value for all temperatures. This treatment does not change in a significative way the $n(T)$ values obtained and therefore we have neglected this approximation.

\section{DISCUSSION}

Three motions have been identified in the present study. The first motion, studied in both copolymers, was observed in the whole temperature range covered. At lower temperatures, where there is not quasielastic broadening, the motion has the character of a pure Debye-Waller vibration. In this range of temperatures the mean-square vibrational amplitude $\left\langle u^{2}\right\rangle$ shows for the lower temperatures the characteristic linear variation of an harmonic behavior. Deviation from linearity at higher temperatures may indicate the appearance of an anharmonic behavior, the onset of a quasielastic component or the entrance of the wing of the inelastic component in the elastic window.

This kind of behavior for $\left\langle u^{2}\right\rangle$ has been observed in a great variety of polymeric and nonpolymeric glass forming materials. ${ }^{22,12}$ The mean-square average displacement $\left\langle u^{2}\right\rangle^{1 / 2}$ measured at the glass transition temperatures $T_{g}$ of the two copolymers gives rise to values of about $0.4 \AA$. This value is comparable to the one observed in polyisobutylene ${ }^{12}$ and smaller than that reported for polybutadiene. ${ }^{29}$ It has been pointed out that the steric hindrance of the motion of the polymeric chain can affect the mean displacement value. In our case, due to the semicrystalline nature of the copolymers, additional restrictions to the dynamics of this motion owing to constrains provoked by the crystalline lamellas cannot be ruled out.

The mode-coupling theory ${ }^{30,31}$ predicts the existence of a temperature $T_{0}$ higher than $T_{g}$ called the dynamical glass transition temperature. This temperature is defined by the long-time behavior of the density correlation function $\Phi_{Q}(t)$, the central quantity of the model coupling theory. More explicitly, one finds

$$
\begin{aligned}
& \Phi_{Q}(t \rightarrow \infty)=0 \text { for } T>T_{0}, \\
& \Phi_{Q}(t \rightarrow \infty)=f_{Q} \text { for } T<T_{0} ;
\end{aligned}
$$

in this context $f_{Q}$, the Debye-Waller factor, is called the nonergodicity parameter or glass form factor. For temperatures lower than, but close to $T_{0}$ one finds the asymptotic law,

$$
f_{Q}=f_{Q}^{c}+h_{Q} \sqrt{T_{0}-T},
$$

where $f_{Q}^{c}$ and $h_{Q}$ are functions of $Q$, which are independent of $T$. In Fig. 15(a) and 15(b) we have represented $\left\langle u^{2}\right\rangle$ as a function of $\left(T_{0}-T\right)^{1 / 2}$ for the heating and cooling cycles of both copolymers. The $T_{0}$ value was introduced in order to have in these plots straight lines. For the copolymers of composition $60 / 40$ and $80 / 20$ we find $T_{0}$ equal to 260 and $270 \mathrm{~K}$, respectively. In both cases $T_{0}$ is $27 \mathrm{~K}$ higher than the corresponding $T_{g}$ value. This kind of fit is one of the best methods to determine $T_{0}$ and this behavior is considered a strong support of the mode-coupling theory of the glass transition. ${ }^{11}$ Buchenau and $\mathrm{Zorn}^{32}$ made an alternative analysis of the $\left\langle u^{2}\right\rangle$ dependence with $T$. They showed that the difference between the experimental results and the extrapolation of the harmonic behavior has an empirical relationship with the viscosity, which allows us to establish a relation between the so-called $\alpha$ and $\beta$ relaxations of the glass transition.

The second motion detected in both copolymers was observed at temperatures around and above $T_{C}$ as a broad quasielastic component in the $\mu \mathrm{eV}$ region. This 

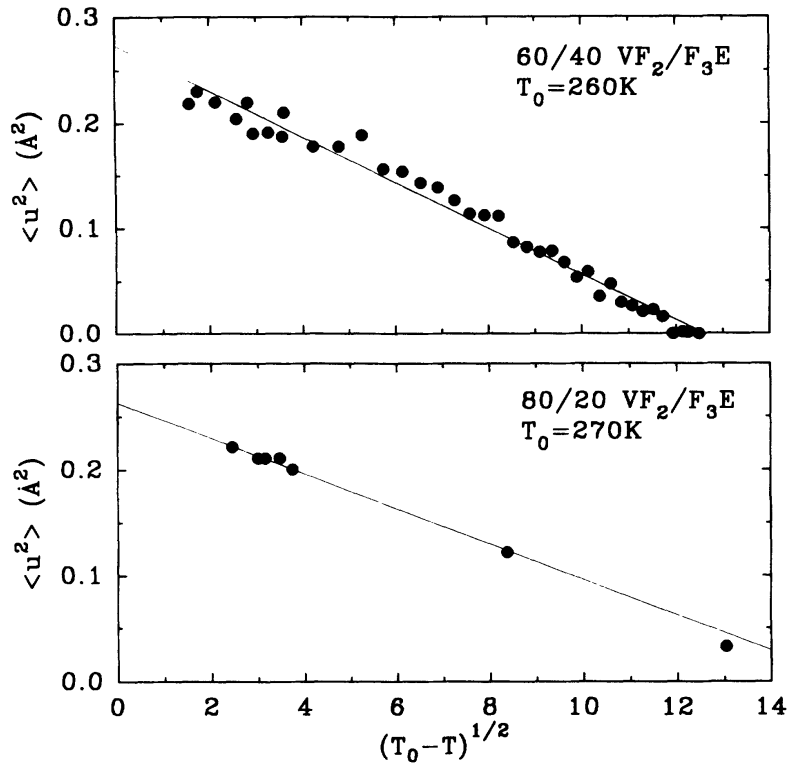

FIG. 15. Displacement $\left\langle u^{2}\right\rangle$ as a function of $\left(T_{0}-T\right)^{1 / 2}$ where $T$ is the temperature and $T_{0}$ the mode-coupling glass transition critical temperature. $T_{0}$ is determined by imposing for these plots a straight line.

motion has been identified as a slow jump diffusive motion of protons attached to each chain in a restricted volume defined by the other chains. This motion has two characteristic geometrical parameters. On one hand, the jump distance $r_{0} \sim 1 \AA$, which has been shown to be almost constant with the temperature and, on the other hand, the length of the available volume, which exhibits a temperature dependence and varies between $L \sim r_{0}$ at $T_{C}=340 \mathrm{~K}$ and $L \sim 5 \AA$ at $T_{m}=423 \mathrm{~K}$ for the $60 / 40$ copolymer. The latter value of $L$ coincides with the distance among adjacent chains as measured by $\mathrm{x}$-ray diffraction. ${ }^{15}$ When this motion is investigated over a broad $Q$ range overoscillations of HWHM, indicating jump anisotropy, are detected (Fig. 10). The jump seems to be favored in a direction that we identify with the chain axis. These results agree with the fact that hydrogens are attached to the polymer chain and therefore obliged to move along the chain axis. At temperatures $T<T_{C}$ the crystalline portion of the copolymer is in the ferroelectric phase with the electric dipoles oriented with absence of motion. The onset of motion appears at $T>T_{C}$ when the crystalline portion of the copolymer becomes paraelectric. The loss of the ferroelectricity may be associated to the onset of this motion. According to the mean-field theory a helpful model in the ferroelectric to paraelectric transition is the "soft-mode" model. In this model it is assumed that the whole spectral response is at a characteristic frequency $\omega_{0}$, so that the phase transition is then an instability of the crystal against this particular mode of deformation of the high-temperature structure. The model also predicts that at $T_{C}$ the frequency of the mode decreases to zero and that the crystal becomes unstable and transforms to a new structure. The observed motion seems to be, in some way, associated to the instability of the crystalline of the crystalline regions in the paraelectric phase.

The third motion was studied in the copolymer $80 / 20$ and appears as a quasielastic broadening in the $\mathrm{meV}$ region. This is a fast local motion of small amplitude $\sim 2 \AA$ restricted to a fraction of the chains. The fraction of protons involved in the motion varies with the temperature. For $T>T_{m}$ all protons and, hence, all chains are involved in the motion. When the temperature decreases in the range $T_{C}<T<T_{m}$ a reduction of the amount of protons involved in this motion is observed. At $T=T_{C}$ a fraction of 0.5 protons is still in motion and, at temperatures below $T_{C}$, a portion of about 0.3 protons remains mobile.

As shown by $x$-ray diffraction ${ }^{18}$ the all-trans conformation of the chains characteristic of the ferroelectric phase transforms to an irregular succession of $\mathrm{tg}^{+}, \mathrm{tg}^{-}$, and $\mathrm{tt}$ sequences in the paraelectric phase. Due to the rotational motion of these sequences, the paraelectric phase has been called the rotator phase. ${ }^{18}$ The above results point out to a combination of a jump diffusion and the rotational motions, with the number of segments involved in the rotation changing from a given value at the Curie temperature to $100 \%$ when the copolymer melts.

Below $T_{C}$ vibrations and the fast local motion were observed. It has been shown by $x$-ray techniques that below $T_{C}$ the crystalline portion has a small amount of nonferroelectric crystalline domains. ${ }^{15}$ From the dynamical point of view in the ferroelectric portion there is absence of motion below $T_{C}$, although in the remaining nonferroelectric domains of the crystalline portion the proton mobility should be allowed. Due to the semicrystalline nature of the investigated samples the contribution of the amorphous portion in the $10^{-11}-10^{-12} \mathrm{~s}$ time scale has to be considered. It has been shown that amorphous polymers exhibits different dynamical processes. The main process, known as $\alpha$ relaxation, which has been detected by several techniques appears as a quasielastic broadening in the $10^{-11}-10^{-8}$ dynamic range at temperatures higher than $T_{g}+50 \mathrm{~K} .{ }^{33} \mathrm{~A}$ faster process has been additionally observed by IQNS techniques (TOF) at time scales $10^{-12}<t<10^{-11} \mathrm{~s}$. Accordingly we have to consider a contribution of the amorphous portion to the observed fast motion at temperatures $T_{g}<T<T_{C}$.

\section{CONCLUSIONS}

We have studied by incoherent quasielastic neutron scattering the molecular dynamics of the ferroelectric copolymers of vinylidene fluoride and trifluoroethylene with two different molar ratios: $80 / 20$ and 60/40. Three motions were identified: (a) The first is a vibrational motion of Debye-Waller type observed in all the temperature range. The low-temperature behavior has been attributed to the amorphous portion and has been analyzed in terms of the mode-coupling theory. (b) The second is a slow jump diffusive motion of each chain in the restricted volume defined by other chains. This motion observed for $T>T_{C}$ is characteristic of the paraelectric phase and 
concerns the crystalline portion of the sample. (c) The third is a fast local small amplitude diffusive motion has been observed at temperatures $T_{g}<T<T_{m}$. The amount of protons involved in this motion raises with temperature exhibiting a dramatical increase for $T>T_{C}$. Different contributions to this motion has been discussed. At $T<T_{C}$ a main contribution of the amorphous portion is expected together with the contribution of some nonferroelectric, though crystalline, domains embedded in the ferroelectric crystalline phase. At $T>T_{C}$ an additional contribution of the crystalline phase owing to the onset of rotational motion in the paraelectric phase is expected.

\section{ACKNOWLEDGMENTS}

Grateful acknowledgments is due to DGICYT (Grant No. PB91-0355), to CICYT (Grant No. MAT92-148), to CICYT (Grant No. MAT94-0740E), to the Consejería de Educación de la Comunidad de Madrid (Grant No. 284/92) and to Fundacion Domingo Martinez (Grant No. $1992 / 93$ ) for the generous support of this investigation.
${ }^{1}$ F. Kremer, S. U. Vallerien, H. Kapitza, R. Zentel, and E. W. Fischer, Phys. Rev. A 42, 3667 (1990).

${ }^{2}$ M. Dumon, H. T. Nguyen, M. Mauzac, C. Destrade, M. F. Achard, and H. Gasparoux, Macromolecules 23, 355 (1990).

${ }^{3}$ T. Furukawa, G. E. Johnson, H. E. Bair, Y. Tajitsu, A. Chiba, and E. Fukada, Ferroelectrics 32, 61 (1981).

${ }^{4}$ K. Tashiro, K. Takano, M. Kobayashi, Y. Chatani, and H. Tadokoro, Ferroelectrics 57, 297 (1984).

${ }^{5}$ T. Yamada and T. Kitayama, J. Appl. Phys. 52, 6859 (1981).

${ }^{6}$ N. Koizumi, N. Haikawa, and H. Habuka, Ferroelectrics 57, 99 (1984).

${ }^{7}$ T. Yagi, M. Tatemoto, and J. Sako, Polym. J. 12, 209 (1980).

${ }^{8}$ A. J. Lovinger, T. Furukawa, G. Davies, and M. G. Broadhurst, Ferroelectrics 50, 227 (1983); Polymer 24, 1225 (1983).

${ }^{9}$ K. Koga and H. Ohigadhi, J. Appl. Phys. 59, 2142 (1986).

${ }^{10}$ J. Colmenero, A. Arbe, and A. Alegria, Phys. Rev. Lett. 71, 2603 (1993).

${ }^{11}$ B. Frick, D. Richter, W. Petry, and U. Buchenau, Z. Phys. B 70, 73 (1988).

${ }^{12}$ B. Frick and D. Richter, Phys. Rev. B 47, 14795 (1993).

${ }^{13}$ J. F. Legrand, Ferroelectrics 91, 303 (1989).

${ }^{14}$ E. López-Cabarcos, A. González Arche, F. Batallán, and B. Frick, Physica B 156-157, 423 (1989).

${ }^{15}$ F. J. Baltá Calleja, A. González Arche, T. A. Ezquerra, C. Santa Cruz, F. Batallán, B. Frick, and E. López Cabarcos, Adv. Polym. Sci. 108, 1 (1993).

${ }^{16}$ Y. Highashihata, J. Sako, and T. Yagi, Ferroelectrics 32, 85 (1981).
${ }^{17}$ T. Furukawa and G. E. Johnson, J. Appl. Phys. 52, 940 (1981),

${ }^{18} \mathrm{~K}$. Tashiro and M. Kobayashi, Polymer 29, 426 (1988).

${ }^{19}$ E. López Cabarcos, A. G. Arche, F. J. Baltá Calleja, and H. G. Zachmann, Makromol. Chem. 20/21, 193 (1988).

${ }^{20}$ E. Lopez Cabarcos, A. G. Arche, F. J. Balta Calleja, P. Bösecke, S. Röber, M. Bark, and H. G. Zachmann, Polymer 32, 3097 (1991).

${ }^{21}$ M. Bee, in Quasielastic Neutron Scattering, edited by Adam Hilger (IOP, Bristol, 1988).

${ }^{22}$ P. G. de Gennes, J. Chem. Phys. 72, 4756 (1980).

${ }^{23}$ J. S. Higgins, R. E. Ghosh, and W. S. Howells, J. Chem. Soc. Faraday Trans. II 73, 40 (1977).

${ }^{24}$ F. Fujara and W. Petry, Europhys. Lett. 4, 921 (1987).

${ }^{25}$ P. A. Egelstaff, An Introduction to the Liquid State (Clarendon, Oxford, 1992).

${ }^{26}$ F. Volino and A. J. Dianoux, Mol. Phys. 41, 271 (1980).

${ }^{27}$ A. J. Dianoux, M. Pineri, and F. Volino, Mol. Phys. 46(1), 129 (1982).

${ }^{28}$ P. L. Hall and D. K. Ross, Mol. Phys. 42, 673 (1981).

${ }^{29}$ T. Kanaya, K. Kaji, and K. Inoue, Macromol. 24, 1826 (1991).

${ }^{30}$ L. Sjögren and W. Götze, in Dynamics of Disordered Materials, Springer Proceedings in Physics, Vol. 37, edited by D. Richter, A. J. Dianoux, W. Petry, and J. Teixeira (SpringerVerlag, Berlin, 1989), p. 18.

${ }^{31}$ W. Götze, Z. Phys. B60, 195 (1985).

${ }^{32}$ U. Buchenau and R. Zorn, Europhys. Lett. 18, 523 (1992).

33 J. Colmenero, A. Alegría, A. Arbe, and B. Frick, Phys. Rev. Lett. 69, 478 (1992). 